\title{
A comparative analysis of clinical characteristics in patients infected with severe acute respiratory syndrome coronavirus 2 (SARS-CoV-2) between Wuhan and Zhoushan, China
}

\author{
Haifeng $\mathrm{Li}^{1}$, Lue $\mathrm{Li}^{1}$, Hanyang Zheng ${ }^{2}$, Mingjiang Xiao ${ }^{2}$, Qiujing Wang ${ }^{3}$, Shibo $\mathrm{Li}^{3}$, Wangyu Zhu ${ }^{4}$ \\ ${ }^{1}$ Respiratory Department, Zhoushan Hospital, Zhoushan, China; ${ }^{2}$ Intensive Care Unit, Zhoushan Hospital, Zhoushan, China; ${ }^{3}$ Department of \\ Infectious Diseases, Zhoushan Hospital, Zhoushan, China; ${ }^{4}$ Cell and Molecular Biology Laboratory, Zhoushan Hospital, Zhoushan, China \\ Contributions: (I) Conception and design: S Li, W Zhu; (II) Administrative support: L Li; (III) Provision of study materials or patients: H Li, H \\ Zheng, M Xiao, Q Wang; (IV) Collection and assembly of data: H Li, H Zheng, M Xiao, Q Wang; (V) Data analysis and interpretation: W Zhu, H \\ Li; (VI) Manuscript writing: All authors; (VII) Final approval of manuscript: All authors. \\ Correspondence to: Shibo Li. Department of Infectious Diseases, Zhoushan Hospital of Zhejiang Province, Zhoushan, Zhejiang 316021 , China. \\ Email: 1sb0398@126.com; Wangyu Zhu. Cell and Molecular Biology Laboratory, Zhoushan Hospital of Zhejiang Province, Zhoushan 316021, \\ China. Email: zhuwangyu24@sina.cn.
}

Background: We aimed to identify any differences in the clinical characteristics of patients treated in Zhoushan Hospital and Wuhan Fourth Hospital, Gutian campus to provide insights into measures to better control the coronavirus disease 2019 (COVID-19) pandemic and treat COVID-19 patients.

Methods: All cases included in this retrospective study from January 10, 2020 to March 15, 2020 were confirmed by laboratory detection of SARS-CoV-2. Data of epidemiological characteristics, clinical characteristics, laboratory results, radiological findings, treatments, and outcomes were obtained from electronic medical records and compared between the patient groups.

Results: A correlation analysis was performed to detect correlations between the serum C-reactive protein (CRP) level and other laboratory findings. COVID-19 patients treated in Wuhan more commonly had fever and shortness of breath, and less commonly had headache compared to those treated in Zhoushan ( $\mathrm{P}=0.002$, 0.039 , and 0.015 , respectively). The period from illness onset to hospitalization in Wuhan was 11.7 7.2 days, which was longer than that in Zhoushan $(4.2 \pm 3.7$ days; $\mathrm{P}=0.002)$, whereas the period from illness onset to shortness of breath in Wuhan was 5.4 \pm 5.0 days, which was shorter than that in Zhoushan $(14.0 \pm 5.6$ days; $\mathrm{P}=0.020)$. Computed tomography scans showed linear opacities, reticulation, and patchy shadows more commonly in cases treated in Wuhan $(\mathrm{P}=0.016,0.013$, and 0.008 , respectively). The mean CRP level in Zhoushan patients was lower than that in Wuhan patients $(\mathrm{P}<0.001)$, and the CRP level was correlated with several laboratory findings related to the immune response.

Conclusions: COVID-19 patients treated at Wuhan Fourth Hospital, Gutian campus had more severe symptoms than those treated at Zhoushan Hospital. Earlier in-hospital treatment, as conducted in Zhoushan, may be beneficial in reducing the severity of illness in COVID-19 patients. Additionally, the correlations between the CRP level and indicators of immune function in COVID-19 patients warrant further investigation.

Keywords: Coronavirus disease 2019 (COVID-19); SARS-CoV-2; pandemic; C-reactive protein (CRP); clinical characteristics

Submitted Oct 19, 2021. Accepted for publication Dec 22, 2021.

doi: 10.21037/apm-21-3629

View this article at: https://dx.doi.org/10.21037/apm-21-3629 


\section{Introduction}

In December 2019, the coronavirus disease 2019 (COVID-19) was outbreak in Wuhan. Although the reported fatality rate of COVID-19 was $4.01 \%$ in China, the total number of deaths has accumulated to 36,797 worldwide as of March 31, which is much higher than the 774 deaths attributed to severe acute respiratory syndrome (SARS) and the 858 deaths attributed to Middle East respiratory syndrome (MERS) (1). Up to now, the epidemic of COVID-19 in China has been effectively controlled through case isolation and contact tracing. Looking forward, a thorough knowledge of the epidemiological and clinical characteristics will help control and treat COVID-19 more effectively.

COVID-19 is caused by severe acute respiratory syndrome coronavirus 2 (SARS-CoV-2) infection, which is a newly identified RNA virus with $\sim 88 \%$ and $~ 80 \%$ sequence similarity to bat SARS-like coronavirus and the SARS virus, respectively, but with only $50 \%$ shared homology with the MERS virus $(2,3)$. Studies have revealed that SARS-CoV-2 binds to angiotensin-converting enzyme 2 (ACE2) expressed on airway epithelial cells and then induces lung injury $(2,4)$ - Several studies have reported differences in COVID-19 outcomes between patients from Wuhan and those from areas outside of Wuhan (5-8). Among the first 41 patients with COVID-19 transferred to Wuhan Jinyintan Hospital, $12(29 \%)$ patients developed acute respiratory distress syndrome (ARDS) and 6 (15\%) died (5). Another report from the same hospital demonstrated that 17 cases $(17 \%)$ progressed to ARDS, and 11 (11\%) patients ultimately died of multiple organ failure (9). Among 138 hospitalized patients in Wuhan Zhongnan Hospital, 27 patients (19.6\%) suffered from ARDS, and 6 (4.3\%) patients died (10). In contrast, Xiao and colleagues reported that only 1 patient developed ARDS among 61 patients infected with SARSCoV-2 in 7 cities of Zhejiang provinces (7). Additionally, among 12 cases of COVID-19 admitted to Shenzhen Third Peoples Hospital, 6 cases (50\%) developed ARDS (11), and in Beijing city, the fatality of patients was $0.9 \%$ (6). Patients with COVID-19 in different areas or cities may have different risks of certain outcomes, and thus, a comparative analysis of the epidemiological and clinical characteristics of patients in different cities may be useful for the control and treatment of COVID-19.

Wuhan is a city in the center of China that is closely connected to 5 provinces, whereas Zhoushan is located in the easternmost region of China on an archipelago. On January 19, 2020, the first case of COVID-19 in Zhoushan was diagnosed at Zhoushan Hospital based on a confirmed laboratory result from the Zhoushan Centers for Disease Control and Prevention (CDC). From then to March 31, 2020 Zhoushan had 10 confirmed cases of COVID-19, and all 10 patients survived and were discharged from Zhoushan Hospital. In the present study, we aimed to identify any differences in the epidemiological and clinical characteristics, including chest computed tomography (CT) and laboratory findings of COVID-19 patients treated in Zhoushan Hospital in Zhoushan city (remote region) and Wuhan Fourth Hospital, Gutian campus in Wuhan (epicenter) to support measures to better control the pandemic and improve the treatment of COVID-19 patients across China and worldwide.

We present the following article in accordance with the STROBE reporting checklist (available at https://dx.doi. org/10.21037/apm-21-3629).

\section{Methods}

\section{Patients and data collection}

A retrospective study was conducted to analyze the differences in epidemiological and clinical characteristics, laboratory findings, treatments, and outcomes between 10 patients with confirmed COVID-19 treated at Zhoushan Hospital in Zhejiang province between January 19 and March 2, 2020 and 102 cases treated in Wuhan Fourth Hospital, Gutian campus in Hubei province between January 10 and March 15, 2020. Both hospitals were designated hospitals for the treatment of COVID-19, and all cases were confirmed as COVID-19 according to the interim guidance of the World Health Organization (WHO) [15] and COVID-19 (trial version 3-7) issued by the China National Health Commission. All cases were confirmed by laboratory detection of SARS-CoV-2. Data and information including epidemiological characteristics, clinical characteristics, laboratory results, and radiological findings as well as treatments and outcomes were obtained from electronic medical records and a simple interview of the patients using standard data collection forms. Information found in the medical records included demographic data, chronic disease and smoking history, symptoms, signs, laboratory findings, CT scans, and treatments with antiviral, including recombinant human interferon $\alpha 2 \mathrm{~b}$, which used to treat with patients in Zhoushan but almost not in Wuhan, antibiotic, and corticosteroid therapy as well as Chinese medicine therapy. The disease onset date was 
the day symptoms were noticed or testing for SARS-CoV-2 was positive in asymptomatic patients. ARDS was diagnosed according to the Berlin definition. The durations of time from illness onset to hospital admission, shortness of breath, and ARDS were also recorded. The last follow-up date was March 30, 2020.

All data were reviewed by 2 doctors who had treated the COVID-19 patients. All procedures performed in this study involving human participants were in accordance with the Declaration of Helsinki (as revised in 2013). The study was approved by the Ethics Review Committee of Zhoushan Hospital (No. 2020(03)), and written informed consent for this retrospective analysis was waived.

\section{Laboratory tests}

At Zhoushan Hospital, sputum and throat swab specimens were collected from the suspected patients, whereas at Wuhan Fourth Hospital, Gutian campus, only throat swab samples were obtained from the patients. The SARS-CoV-2 nucleotide sequence was detected in all samples by real-time reverse transcription polymerase chain reaction analysis, and testing was repeated with a new specimen 24 hours later. Other laboratory tests, such as blood count, C-reactive protein (CRP), serum biochemistry, D-dimer, and others, were conducted on the day of hospital admission.

\section{Statistical analysis}

Data of continuous variables are presented as medians with interquartile ranges (IQRs) or means and standard deviations according to the normal distribution test. The Mann-Whitney test or two-tailed $t$-test was used to compare data between the two groups, and the frequency data of the two groups were compared using the Chi-square or Fisher's exact test. The Spearman correlation test was conducted to evaluate the relationship between CRP level and other laboratory findings. All statistical analyses were performed using SPSS software version 17.0 (SPSS, Inc.) and GraphPad Prism version 5.00 software (GraphPad Software, Inc.), and differences for which the $\mathrm{P}$ value was no greater than 0.05 were considered statistically significant.

\section{Results}

\section{Epidemiological features and clinical characteristics}

By March 24, 2020, a total of 112 patients with COVID-19 were enrolled in the present study, including 10 cases treated in Zhoushan Hospital and 102 treated in Wuhan Fourth Hospital, Gutian campus. Among the 10 cases treated in Zhoushan Hospital: 3 were from the same institution and had become infected with SARS-CoV-2 after visiting friends from Wuhan who were then confirmed to be infected with SARS-CoV-2 in Wuhan; 2 were from Wuhan and were already infected when they came to visit their son in Zhoushan; 2 were Zhoushan residents who had gone to Wuhan on business and became infected with the virus before returning to Zhoushan; and the last 3 patients were exposed when a relative with confirmed COVID-19 came from Wuxi, Jiangsu Province to visit their family. The detailed epidemiological features of the cases treated in Wuhan were unclear as no traceable sources of infection were known. All included cases treated in Wuhan were considered community acquired infection.

The demographic and clinical features of the included patients are described in Table 1. Among the 10 patients treated in Zhoushan, the median age was 52 years, and none were current smokers. Three $(30 \%)$ of the patients in Zhoushan had 1 or more underlying comorbidities, 2 had hypertension, and 2 had diabetes. Among the 102 cases treated in Wuhan, the median age was 61 years, and 17 (16.7\%) cases were current smokers. Overall, 55 (53.9\%) cases had underlying diseases, including $9(8.8 \%)$ with hypertension, $13(12.7 \%)$ with diabetes, 10 (9.8\%) with hypertension and diabetes, 5 (4.9\%) with renal disease, 5 (4.9\%) with cerebrovascular disease, 4 (3.9\%) with cardiac disease, $4(3.9 \%)$ with a malignant tumor after surgery, 2 (2.0\%) with chronic obstructive pulmonary disease, 2 (2.0\%) with psychosis, and $1(1.0 \%)$ with gastrointestinal bleeding. The age, sex, smoking status, and incidence of underlying comorbidities were comparable between cases treated in Zhoushan and Wuhan (all $\mathrm{P}>0.05$ ).

Among all patients, the most common symptoms of COVID-19 were fever (96.4\%), followed by cough (87.55\%), shortness of breath $(62.5 \%)$, fatigue $(61.6 \%)$, and diarrhea $(32.1 \%)$. Patients treated in Wuhan more commonly had fever and shortness of breath and less commonly had headache compared to the cases treated in Zhoushan ( $\mathrm{P}=0.002,0.039$, and 0.015 , respectively). The period from illness onset to hospitalization for Wuhan patients was $11.7 \pm 7.2$ days, which was longer than that for Zhoushan patients $(4.2 \pm 3.7$ days; $\mathrm{P}=0.002)$, whereas the period from illness onset to shortness of breath for Wuhan patients was 5.4 \pm 5.0 days, which was shorter than that for Zhoushan patients $(14.0 \pm 5.6$ days; $\mathrm{P}=0.020)$. However, the 
Table 1 Comparison of clinical features of 112 hospitalized COVID-19 patients treated in Zhoushan and Wuhan, n (\%)

\begin{tabular}{|c|c|c|c|c|}
\hline Characteristics & Total $(\mathrm{N}=112)$ & Zhoushan $(n=10)$ & Wuhan $(n=102)$ & $P$ value \\
\hline$\leq 60$ & $46(41.1)$ & $6(60.0)$ & $40(39.2)$ & 0.313 \\
\hline$>60$ & $66(58.9)$ & $4(40.0)$ & $62(60.8)$ & \\
\hline \multicolumn{5}{|l|}{ Sex } \\
\hline Male & $51(45.5)$ & $7(70.0)$ & $44(43.1)$ & \\
\hline \multicolumn{5}{|l|}{ Smoking } \\
\hline Non-smoking & $95(84.8)$ & $10(100.0)$ & 85 (83.3) & 0.222 \\
\hline Current and ever smoking & $17(15.2)$ & 0 & $17(16.7)$ & \\
\hline \multicolumn{5}{|l|}{ Symptoms } \\
\hline Fever & $108(96.4)$ & $7(70.0)$ & $101(99.0)$ & 0.002 \\
\hline Highest temperature, ${ }^{\circ} \mathrm{C}$ & $38.2 \pm 3.67$ & $38.2 \pm 1.08$ & $38.2 \pm 3.86$ & 0.991 \\
\hline \multicolumn{5}{|l|}{ Cough type } \\
\hline Dry cough & $61(54.5)$ & $6(60.0)$ & $55(53.9)$ & 1.000 \\
\hline Cough with sputum & $37(33.0)$ & $3(30.0)$ & 34 (33.3) & \\
\hline No cough & $14(12.5)$ & $1(10.0)$ & $13(12.7)$ & \\
\hline Fatigue & $69(61.6)$ & $6(60.0)$ & $63(61.8)$ & 1.000 \\
\hline Sore throat & $11(9.8)$ & $1(10.0)$ & $10(9.8)$ & 1.000 \\
\hline Diarrhea & $36(32.1)$ & $2(20.0)$ & $34(33.3)$ & 0.497 \\
\hline Nausea and vomiting & $16(14.3)$ & $2(20.0)$ & $14(13.7)$ & 0.633 \\
\hline Chest pain & $4(3.6)$ & $1(10.0)$ & $3(2.9)$ & 0.316 \\
\hline Arrhythmia & $7(6.3)$ & $1(10.0)$ & $6(5.9)$ & 1.000 \\
\hline Days from illness onset to hospitalization & $11.1 \pm 7.3$ & $4.2 \pm 3.7$ & $11.7 \pm 7.2$ & 0.002 \\
\hline Days from illness onset to shortness of breath & $5.6 \pm 5.2$ & $14.0 \pm 5.6$ & $5.4 \pm 5.0$ & 0.020 \\
\hline Hospital stay & $16.6 \pm 7.7$ & $20.0 \pm 9.4$ & $16.2 \pm 7.4$ & 0.136 \\
\hline \multicolumn{5}{|l|}{ Comorbid conditions } \\
\hline ARDS & $7(6.3)$ & $1(10.0)$ & $6(5.9)$ & 1.000 \\
\hline Shock & $2(1.8)$ & 0 & $2(2.0)$ & 1.000 \\
\hline
\end{tabular}

Table 1 (continued) 
Table 1 (continued)

\begin{tabular}{lcccc}
\hline Characteristics & Total $(\mathrm{N}=112)$ & Zhoushan $(\mathrm{n}=10)$ & Wuhan $(\mathrm{n}=102)$ & $\mathrm{P}$ value \\
\hline Treatments & & & & \\
Antiviral treatment & $108(96.4)$ & $10(100.0)$ & $98(96.1)$ & 1.000 \\
Interferon alpha inhalation & $10(8.9)$ & $10(100.0)$ & 0 & $<0.001$ \\
Antibiotic treatment & $83(74.1)$ & $4(40.0)$ & $79(77.5)$ & 0.018 \\
Corticosteroid and gamma globulin & $31(27.7)$ & $2(20.0)$ & $29(28.4)$ & 0.724 \\
Thymosin & $5(4.5)$ & $1(10.0)$ & $4(3.9)$ & 0.374 \\
Proprietary Chinese medicine & $53(47.3)$ & $1(10.0)$ & $52(51.0)$ & 0.018 \\
Subtypes & & & & 0.452 \\
Common-type & $85(75.9)$ & $7(70.0)$ & $78(76.5)$ & $18(17.6)$ \\
Severe-type & $21(18.8)$ & $3(30.0)$ & $6(5.9)$ & 0.512 \\
Fatal-type & $6(5.4)$ & 0 & $3(2.9)$ \\
Outcome & & & $83(81.4)$ \\
Death & $3(2.7)$ & $10(100.0)$ & $16(15.7)$ \\
Discharged & $93(83.0)$ & 0 & \\
Continued hospitalization & $16(14.3)$ & & \\
\hline
\end{tabular}

length of hospital stay for patients was comparable between patients treated in the two cities $(\mathrm{P}=0.136)$. Patients treated in Zhoushan were more likely to receive interferon alpha inhalation therapy compared with patients treated in Wuhan $(\mathrm{P}<0.001)$, whereas patients treated in Wuhan were more likely to receive antibiotic therapy $(\mathrm{P}=0.018)$ and the proprietary Chinese medicine lianhua qingwen capsule $(\mathrm{P}=0.018)$. The subtypes and outcomes were comparable between the two cities $(\mathrm{P}=0.452$ and 0.512 , respectively). Only 1 patient treated in Wuhan was transferred to the intensive care unit (ICU) and then died.

\section{CT and laboratory findings}

All COVID-19 cases treated in Zhoushan and Wuhan underwent CT scanning and blood tests on hospital admission. Most cases (108/112, 96.4\%) showed typical CT findings with ground-glass opacities with or without consolidation (Table 2; Figures 1,2). Linear opacities, reticulation, and patchy shadows were observed more commonly in cases treated in Wuhan $(\mathrm{P}=0.016,0.013$, and 0.008 , respectively). The blood cell test results demonstrated that the CRP level was lower in patients treated in Zhoushan than that in patients treated in Wuhan
$(\mathrm{P}<0.001)$, whereas the monocyte count, hemoglobin $(\mathrm{Hb})$ level, and serum albumin (ALB) level were lower in patients treated in Wuhan than in those treated in Zhoushan $(\mathrm{P}=0.038,0.001$, and $<0.001$, respectively). Meanwhile, the serum level of creatine kinase (CK)-MB was higher in patients treated in Wuhan than those treated in Zhoushan $(\mathrm{P}<0.001)$.

\section{Correlation of CRP with other laboratory findings}

The serum level of CRP is reportedly higher in severe and fatal cases of COVID-19 (12-14), and in the present study, we found that the level of CRP was increased in patients treated in Wuhan. Thus, we further analyzed the potential correlations of serum CRP level with other laboratory findings. The results revealed that the serum level of CRP was positively correlated with the circulating neutrophil count $(t=0.290, P=0.002)$, CK-MB level $(t=0.326, P=0.003)$, blood urea nitrogen (BUN) level $(t=0.363, \mathrm{P}=0.007)$, lactate dehydrogenase $(\mathrm{LDH})$ level $(t=0.561, \mathrm{P}<0.001)$, and $\mathrm{D}$-dimer level $(t=0.204, \mathrm{P}=0.035)$, and was negatively associated with the circulating lymphocyte count $(t=-0.534$, $\mathrm{P}<0.001)$, monocyte count $(t=-0.249, \mathrm{P}=0.008)$, eosinophil count $(t=-0.398, \mathrm{P}<0.001)$, platelet count $(t=-0.246$, 
Table 2 Comparison of CT and laboratory findings for 112 hospitalized COVID-19 patients treated in Zhoushan and Wuhan, n (\%) or medians (IQRs)

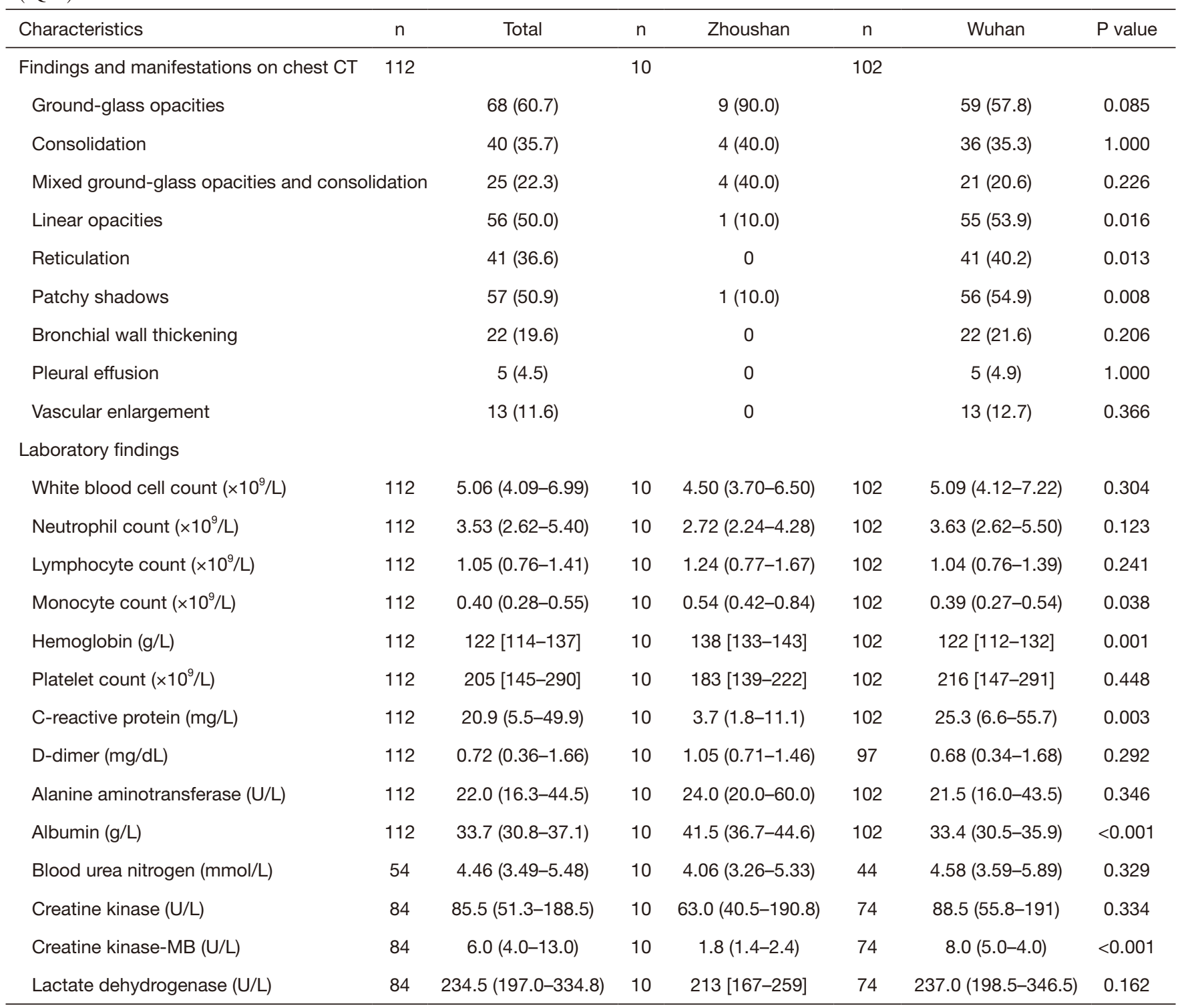

$\mathrm{P}=0.009)$, and ALB level ( $t=-0.505, \mathrm{P}<0.001$, Figure 3). Other laboratory results such as white blood cell count, $\mathrm{Hb}$ level, alanine aminotransferase (ALT) level, and CK level showed no correlation with the CRP level (all $\mathrm{P}<0.05$ ).

\section{Discussion}

COVID-19 has become a unique international pandemic in human history and may become the first controlled pandemic. A clear understanding of the differences in the epidemiological and clinical characteristics as well as treatment approaches applied in different patient populations will contribute to better control of the pandemic. We report here that two COVID-19 patient cohorts from Zhoushan Hospital and Wuhan Fourth Hospital, Gutian campus had different epidemiological and clinical features. Notably, the close contacts could be traced in all cases treated in Zhoushan, whereas the patients treated in Wuhan had community acquired infection and hard to trace. Moreover, differences in clinical features, CT findings, and laboratory results were observed between the patient groups. 

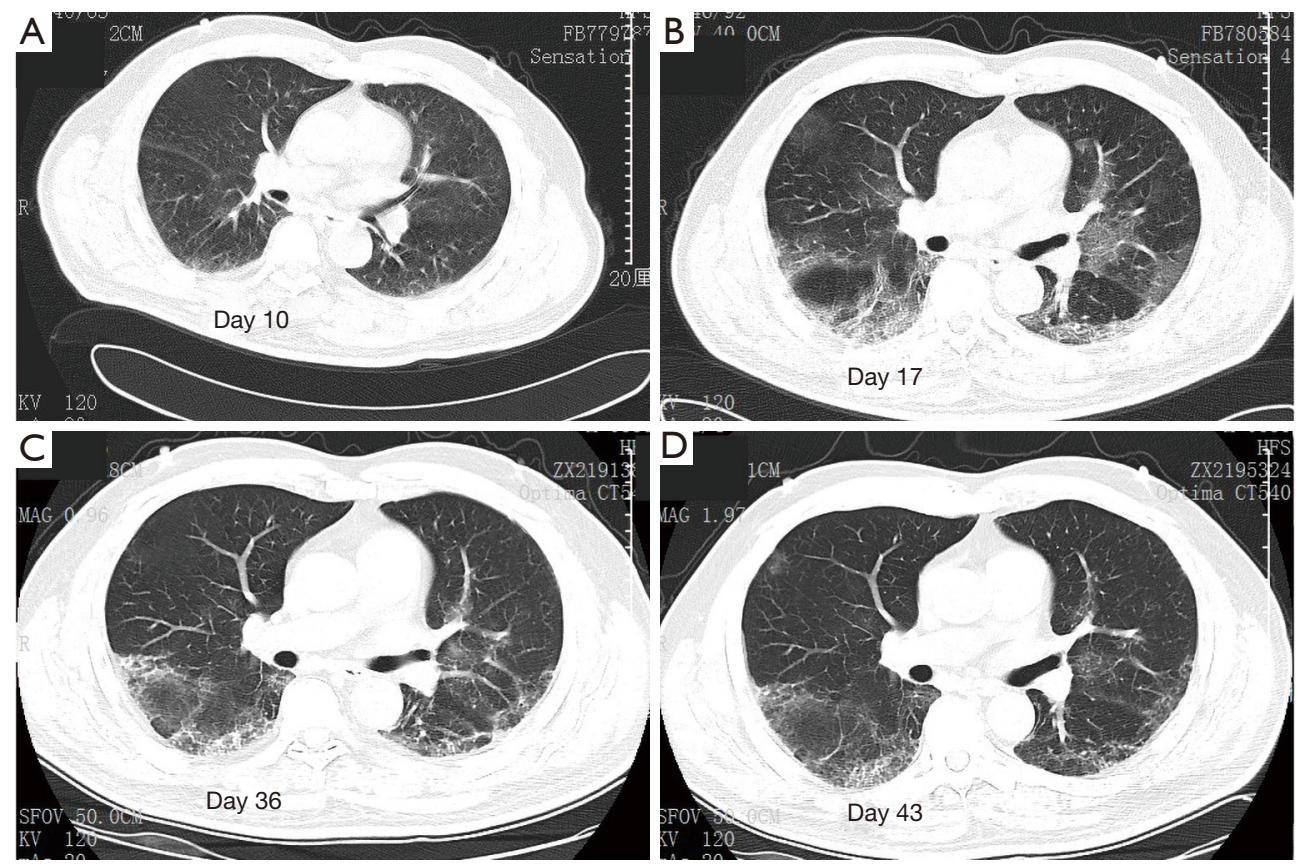

Figure 1 CT images of a 66-year-old man without a smoking history treated in Zhoushan. (A) Day 10 from illness onset: small amount of patchy shadows observed bilaterally near the pleura; (B) Day 17: mixed ground-glass opacities scattered with an unclear boundary observed bilaterally; (C) Day 36: patchy shadows with linear opacities and reticulation observed bilaterally; (D) Day 43: lesions appear further absorbed. The patient was discharged to home 1 day after the last scan. CT, computed tomography.

The first case of COVID-19 in Zhoushan was diagnosed on January 20, 2020, and beginning 3 days later, all people entering Zhoushan had to be screened for potential infection. As an island city, it was relatively easy for Zhoushan to control the passage of individuals across intercity channels. Meanwhile, the Zhoushan CDC followed all suspected cases and the close contacts of the confirmed and suspected cases as soon as possible. Moreover, the community staff checked whether citizens had traveled to Wuhan, Hubei Province or other cities that had a certain number of patients. With these measures in place, Zhoushan had only 10 cases of COVID-19 during the study period. In contrast, many cases in Wuhan were considered community acquired infections, and on January 23, 2020, Wuhan was sealed off from outside contact to inhibit the spread of SARS-CoV-2. Fourteen days later, the epidemic of COVID-19 was considered to be significantly controlled in China. The similar symptoms of patients in the two cohorts in the present study included the common signs of fever, cough, shortness of breath, fatigue, and diarrhea. However, cases treated in Wuhan more commonly had fever and shortness of breath than those treated in Zhoushan.
This may have been because patients in Zhoushan received high-quality in-hospital care soon before the onset of severe symptoms. Because the number of suspected cases in Wuhan was so high, some cases could not be detected early, and some patients had to wait for empty beds to become available before they could be hospitalized. However, patients in Zhoushan were detected in early time by tracking of CDC and hospitalized soon. Notably, the percentage of patients with shortness of breath was higher among the patients treated in Wuhan than among those treated in Zhoushan. Because some mild cases in Wuhan could not be hospitalized before progression to common-type or severe-type, the period from illness onset to hospitalization was longer in Wuhan than in Zhoushan $(11.7 \pm 7.2 v s$. 4.2 \pm 3.7 days). In Beijing, the reported period from illness onset to hospital presentation was $4.5 \pm 3.7$ days (6). Conversely, the period from illness onset to shortness of breath in Wuhan was $5.4 \pm 5.0$ days, which was shorter than that in Zhoushan (14.0 \pm 5.6 days). A study from Zhejiang Province also reported that the symptoms which persisted for longer than 10 days in Wuhan were more severe than those in cases in Zhejiang (5,7). Notably, although 3 patients 

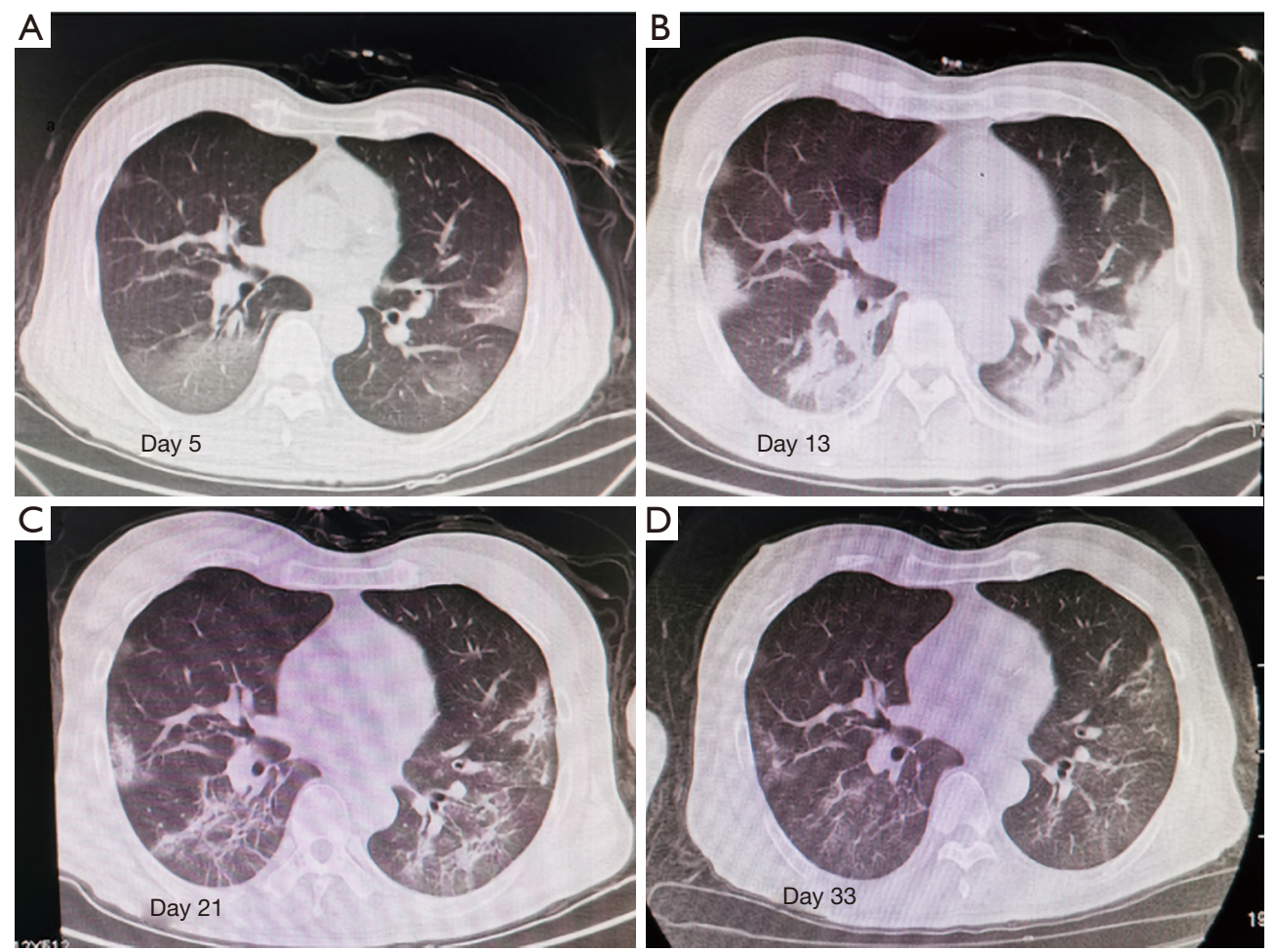

Figure 2 CT images of a 48-year-old man without a smoking history treated in Wuhan. (A) Day 5 from illness onset: multiple ground-glass opacities observed near the pleura; (B) Day 13: lesions appear dense with consolidation and air bronchogram; (C) Day 21: lesions show some absorption with irregular linear opacities and reticulation; (D) Day 33: lesions appear further dissipated. The patient was discharged from the hospital 3 days after the last scan. CT, computed tomography.

in our Wuhan cohort died, the clinical outcomes of the 2 cohorts showed no statistically significant differences. Thus, larger cohort sizes are needed to better analyze potential differences in outcomes between patients treated in these 2 locations.

Patients in both cohorts received antiviral therapy with lopinavir and ritonavir, and severe-type and fatal cases received corticosteroid therapy. Only 4 cases $(40.0 \%)$ in Zhoushan were treated with antibiotic therapy, whereas 79 cases $(77.5 \%)$ in Wuhan received antibiotic therapy, which is similar to the percentages in other studies $(5,9)$. Patients in Wuhan may have had a greater risk for co-infection with bacteria, and thus, their doctors tended to administer antibiotic therapy to reduce the potential for complications. The proprietary Chinese medicine lianhua qingwen capsule was also used more commonly to treat patients in Wuhan for its apparent antiviral effect. Patients treated in Zhoushan received interferon alpha inhalation, whereas those treated in Wuhan did not. Because we did not observe a statistically significant difference in outcomes between our patient cohorts, we cannot speculate on which therapies may have been more effective. We believe interferon alpha inhalation and the proprietary Chinese medicine lianhua qingwen capsule both had antiviral effects and all patients received adequate therapy in the hospitals (15).

The typical CT findings for patients with COVID-19 are consistent with those for viral pneumonia with ground-glass opacities or mixed ground-glass opacities and consolidation $(16,17)$. Patients in Wuhan had more severe and late-stage CT findings than those in Zhoushan, with linear opacities, reticulation, and patchy shadows. This may because the time from onset to hospitalization was longer in Wuhan than that in Zhoushan. With regard to the laboratory findings, the serum CRP levels in patients treated in Wuhan were much higher than those in patients treated in Zhoushan, suggesting that the patients in Wuhan may have been coinfected with bacteria. Moreover, the $\mathrm{Hb}$ and ALB levels were lower and the CK-MB level was higher in patients in Wuhan, and these differences may have been attributable to the higher prevalence of underlying diseases, such as 


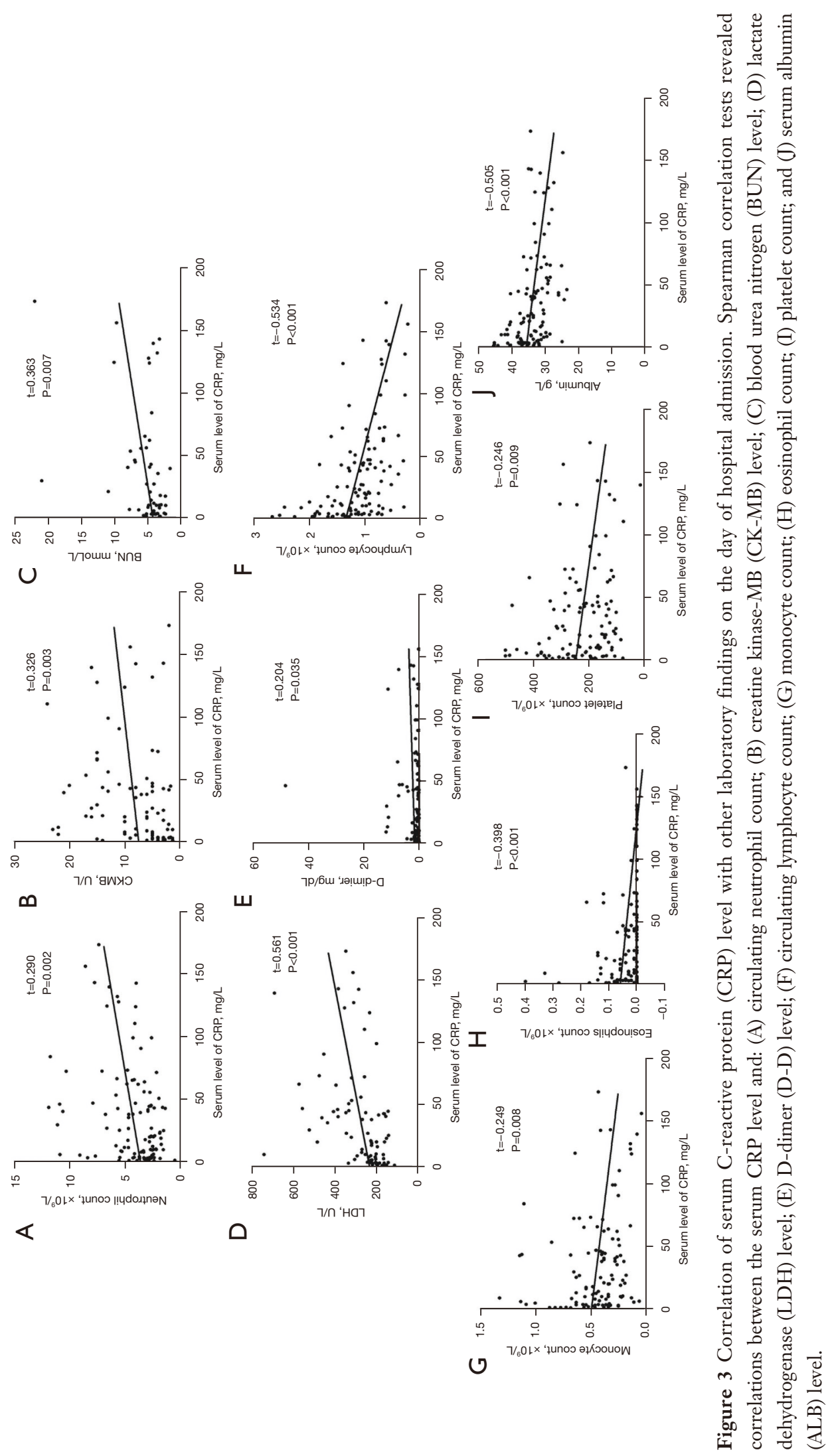


gastrointestinal bleeding, uremia, and cardiac disease, as well as the more severe condition of the patients. The latter cause is supported by the previous finding that nonICU cases had higher $\mathrm{Hb}$ levels than ICU cases (18). Interestingly, our results showed that the CRP level was positively correlated with the circulating neutrophil count and negatively associated with the circulating lymphocyte, monocyte, eosinophil, and platelet counts, and thus may reflect the ability of patients' immune systems to resist SARS-CoV-2. This possibility warrants further investigation.

The present study has several limitations. First, only 10 patients were enrolled from Zhoushan Hospital, and thus, there was bias in the study population. Second, the study included only patients treated in 2 hospitals, one in each city, and analysis of more cases treated in more hospitals in each city could provide greater insight into differences in clinical features, CT findings, and laboratory results. Third, at the last follow-up, 19 patients remained hospitalized, and thus, analysis of outcomes in these cases was not possible.

In conclusion, patients treated in Wuhan Fourth Hospital, Gutian campus had more severe symptoms than those treated in Zhoushan Hospital with a longer period from illness onset to hospitalization and a shorter period from illness onset to shortness of breath. An effective treatment regimen for COVID-19 remains to be discovered. However, the earlier in-hospital treatment of patients in Zhoushan may have helped to reduce the severity of COVID-19 in these patients. Finally, further analysis of the association between serum CRP levels and markers of immune response in COVID-19 patients is warranted.

\section{Acknowledgments}

We thank all the patients included in this study and Wuhan Fourth Hospital for the provision of the cases and the collaboration in the treatment of patients.

Funding: This study was supported by the Health High Level Talents of Zhejiang Province and the Science and Technology Bureau of Zhoushan (Nos. 2020C31001, 2020C31002, 2020C31003).

\section{Footnote}

Reporting Checklist: The authors have completed the STROBE reporting checklist. Available at https://dx.doi. org/10.21037/apm-21-3629

Data Sharing Statement: Available at https://dx.doi. org/10.21037/apm-21-3629

Conflicts of Interest: All authors have completed the ICMJE uniform disclosure form (available at https://dx.doi. org/10.21037/apm-21-3629). The authors have no conflicts of interest to declare.

Ethical Statement: The authors are accountable for all aspects of the work in ensuring that questions related to the accuracy or integrity of any part of the work are appropriately investigated and resolved. All procedures performed in this study involving human participants were in accordance with the Declaration of Helsinki (as revised in 2013). The study was approved by the Ethics Review Committee of Zhoushan Hospital (No. 2020(03)), and written informed consent for this retrospective analysis was waived.

Open Access Statement: This is an Open Access article distributed in accordance with the Creative Commons Attribution-NonCommercial-NoDerivs 4.0 International License (CC BY-NC-ND 4.0), which permits the noncommercial replication and distribution of the article with the strict proviso that no changes or edits are made and the original work is properly cited (including links to both the formal publication through the relevant DOI and the license). See: https://creativecommons.org/licenses/by-nc-nd/4.0/.

\section{References}

1. Mahase E. Coronavirus covid-19 has killed more people than SARS and MERS combined, despite lower case fatality rate. BMJ 2020;368:m641.

2. Ahmed SF, Quadeer AA, McKay MR. Preliminary Identification of Potential Vaccine Targets for the COVID-19 Coronavirus (SARS-CoV-2) Based on SARSCoV Immunological Studies. Viruses 2020;12:254.

3. Chan JF, Kok KH, Zhu Z, et al. Genomic characterization of the 2019 novel human-pathogenic coronavirus isolated from a patient with atypical pneumonia after visiting Wuhan. Emerg Microbes Infect 2020;9:221-36.

4. Lu R, Zhao X, Li J, et al. Genomic characterisation and epidemiology of 2019 novel coronavirus: implications for virus origins and receptor binding. Lancet 2020;395:565-74.

5. Huang C, Wang Y, Li X, et al. Clinical features of patients infected with 2019 novel coronavirus in Wuhan, China. Lancet 2020;395:497-506.

6. Tian S, Hu N, Lou J, et al. Characteristics of COVID-19 
infection in Beijing. J Infect 2020;80:401-6.

7. $\mathrm{Xu} X W, \mathrm{Wu} X X$, Jiang $\mathrm{XG}$, et al. Clinical findings in a group of patients infected with the 2019 novel coronavirus (SARS-Cov-2) outside of Wuhan, China: retrospective case series. BMJ 2020;368:m606.

8. Zhang JJ, Dong X, Cao YY, et al. Clinical characteristics of 140 patients infected with SARS-CoV-2 in Wuhan, China. Allergy 2020;75:1730-41.

9. Chen N, Zhou M, Dong X, et al. Epidemiological and clinical characteristics of 99 cases of 2019 novel coronavirus pneumonia in Wuhan, China: a descriptive study. Lancet 2020;395:507-13.

10. Wang D, Hu B, Hu C, et al. Clinical Characteristics of 138 Hospitalized Patients With 2019 Novel CoronavirusInfected Pneumonia in Wuhan, China. JAMA 2020;323:1061-9.

11. Liu Y, Yang Y, Zhang C, et al. Clinical and biochemical indexes from 2019-nCoV infected patients linked to viral loads and lung injury. Sci China Life Sci 2020;63:364-74.

12. Chen G, Wu D, Guo W, et al. Clinical and immunological features of severe and moderate coronavirus disease 2019. J Clin Invest 2020;130:2620-9.

Cite this article as: $\mathrm{Li} \mathrm{H}$, Li L, Zheng H, Xiao M, Wang Q, Li S, Zhu W. A comparative analysis of clinical characteristics in patients infected with severe acute respiratory syndrome coronavirus 2 (SARS-CoV-2) between Wuhan and Zhoushan, China. Ann Palliat Med 2021;10(12):12810-12820. doi: 10.21037/apm-21-3629
13. Gao Y, Li T, Han M, et al. Diagnostic utility of clinical laboratory data determinations for patients with the severe COVID-19. J Med Virol 2020;92:791-6.

14. Wang L. C-reactive protein levels in the early stage of COVID-19. Med Mal Infect 2020;50:332-4.

15. Luo H, Tang QL, Shang YX, et al. Can Chinese Medicine Be Used for Prevention of Corona Virus Disease 2019 (COVID-19)? A Review of Historical Classics, Research Evidence and Current Prevention Programs. Chin J Integr Med 2020;26:243-50.

16. Shi $H$, Han $X$, Jiang N, et al. Radiological findings from 81 patients with COVID-19 pneumonia in Wuhan, China: a descriptive study. Lancet Infect Dis 2020;20:425-34.

17. Xie X, Zhong Z, Zhao W, et al. Chest CT for Typical Coronavirus Disease 2019 (COVID-19) Pneumonia: Relationship to Negative RT-PCR Testing. Radiology 2020;296:E41-5.

18. Fan BE, Chong VCL, Chan SSW, et al. Hematologic parameters in patients with COVID-19 infection. Am J Hematol 2020;95:E131-4.

(English Language Editor: C. Betlzar) 